\title{
Reconstructive rhinoplasty with costal cartilage grafting: A case report of relapsing polychondritis
}

Yunhae Lee, Hyungon Choi

Department of Plastic and

Reconstructive Surgery, Konkuk

University Medical Center, Seoul, Korea

\begin{abstract}
Relapsing polychondritis (RP) is a rare autoimmune disorder of unknown etiology characterized by recurrent episodes of inflammation and the destruction of cartilaginous tissues, primarily involving the ear, nose, and the respiratory tract. Nasal chondritis is present in $24 \%$ of patients at the time of diagnosis and develops subsequently in $53 \%$ throughout the diseases progress. Progressive destruction of nasal cartilage leads to the characteristic flattening of the nasal bridge, resulting in the saddle nose deformity. In patients with RP, surgical management for saddle nose is carefully decided due to the disease relapsing characteristics. We present a RP patient with a saddle nose deformity who underwent reconstruction rhinoplasty with autologous costal cartilage grafting. At 6-month follow-up, the patient retained good esthetic results and showed neither complication nor relapse of RP.
\end{abstract}

Keywords: Costal cartilage / Polychondritis, relapsing / Rhinoplasty

\section{INTRODUCTION}

Relapsing polychondritis (RP) is a rare autoimmune disorder of unknown etiology that primarily affects cartilaginous tissues [1]. RP is characterized by recurrent episodes of inflammation, that causes progressive anatomical deformation and functional impairment of primarily the auricular cartilage, the nasal cartilage, and the respiratory tract [1,2]. McAdam et al. [3] proposed that RP can be diagnosed when three or more of these features are present, along with a positive biopsy from the affected tissue: (1) recurrent chondritis of both auricles; (2) nonerosive inflammatory polyarthritis; (3) chondritis of nasal cartilage; (4) inflammation of ocular structures, including conjunctivitis, keratitis, scleritis, episcleritis, and uveitis; (5) chondritis in the respiratory tract involving laryngeal and/or tracheal cartilages; and (6) cochlear and/or vestibular damage manifesting as sensorineural hearing loss, tinnitus, or vertigo. Damiani and

Correspondence: Hyungon Choi

Department of Plastic and Reconstructive Surgery, Konkuk University Medical

Center, 120-1 Neungdong-ro, Gwangjin-gu, Seoul 05030, Korea

E-mail: 20040059@kuh.ac.kr

Received September 3, 2019 / Revised October 1, 2019 / Accepted October 2, 2019
Levine modified this criteria as followed: (1) at least three or more of McAdam's diagnostic features with or without histologic findings; (2) one or more of McAdam's diagnostic features with histologic findings of RP; and (3) chondritis in two or more separate locations with response to steroids and/or Dapson [4]. Nasal chondritis is present in $24 \%$ of patients at the time of diagnosis and develops subsequently in 53\% throughout the disease's progress [5]. Progressive nasal chondritis leads to the flattening of the nasal bridge, resulting in the painless irreversible saddle nose deformity [1]. However, the number of patients treated for saddle nose deformity remains limited due to the small number of patients reported and the case reports are heterogeneous with different outcomes [1]. Here, we present a case of a RP patient treated at our department to correct his saddle nose deformity. Our patient underwent costal cartilage grafting and attained good results with neither relapse of nasal chondritis nor donor site problems.

\section{CASE REPORT}

A 35-year-old man was diagnosed with RP at another hospital 
after uncontrolled (showing resistance to antibiotics) pain and swelling in his right ear. During the patient's initial visit to Konkuk University Medical Center at January 2007 with a chief complaint of hoarseness, the patient was under oral administration of prednisolone (PSL) to $17.5 \mathrm{mg} /$ day and showed severe saddle nose deformity and deformity of the both external ears (Fig. 1). The patient's non-enhanced chest computer tomography showed diffuse thickening of the proximal trachea and mild narrowing at expiration (Fig. 2). The patient was diagnosed with acute labyrinthitis after complaining of otalgia and dizziness. As the patient showed four features of McAdam's diagnostic criteria for RP, we re-confirmed the diagnosis and increased the oral administration of PSL to $40 \mathrm{mg}$ /day. Also after plasmapheresis, erythrocyte sedimentation rate dropped down to 2 from $68 \mathrm{~mm} / \mathrm{hr}$. The patient was referred to our department on August 2010, after cessation of PSL treatment for 3 months. As the patient showed symptoms under control for over 15 months with a PSL maintenance dose of $5 \mathrm{mg}$ /day and no signs of aggravation without PSL for 3 months, we planned surgery to correct his saddle nose deformity with costal cartilage grafting.

An open rhinoplasty technique was used and after dissection, blunting of the anterior septal angle and destruction of the upper lateral cartilage were obvious. A portion of the 8th costal cartilage was harvested and carved with a blade to mimic a silicone implant (Fig. 3). The carved cartilage fragment was inserted into the subperiosteal pocket of the nasal dorsum. The remaining costal cartilage was used to support the columella as a strut graft (Fig. 4).

The patient showed good esthetic results with neither complication nor relapse of RP for 6 months after surgery (Fig. 5). Two episodes of RP recurrence occurred after surgery (15 months
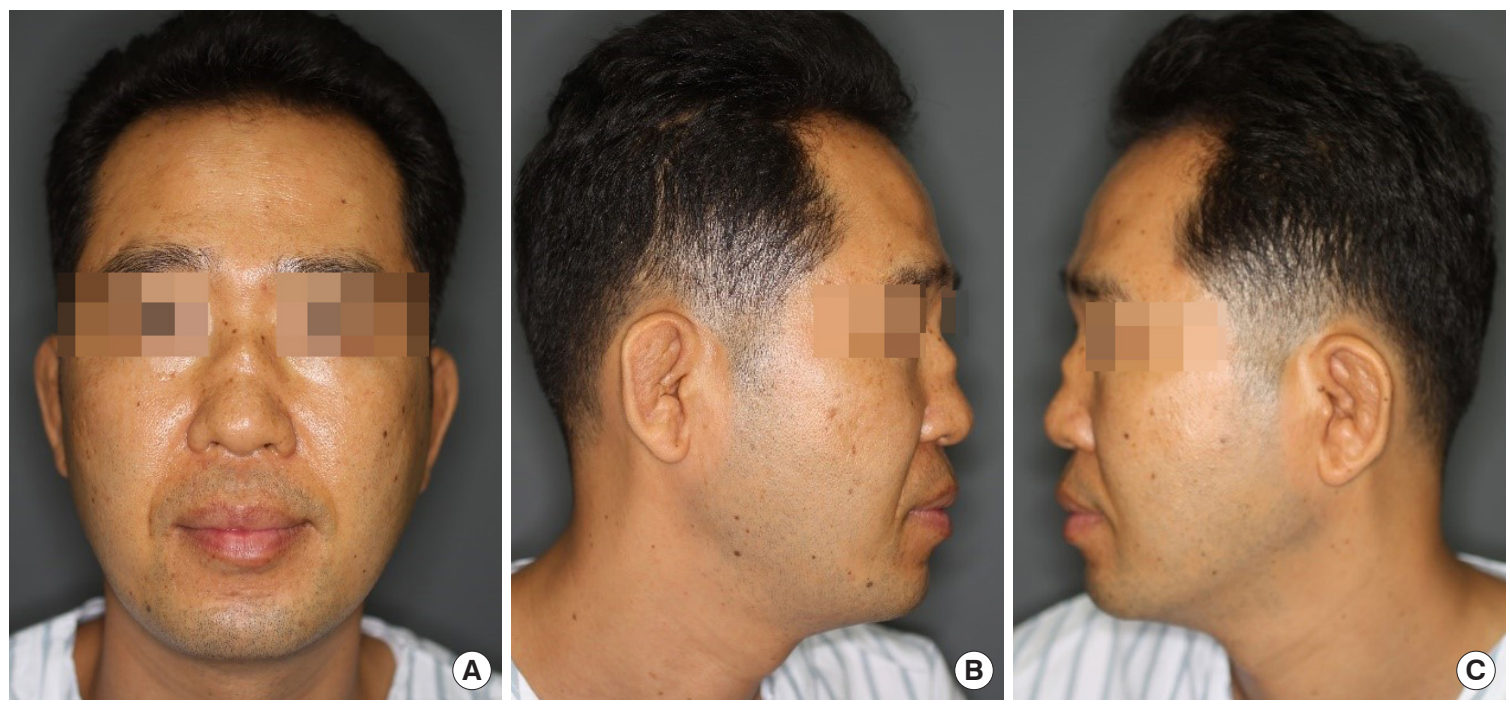

Fig. 1. Preoperative photographs of the patient. (A) Saddle nose deformity, anteroposterior view. (B) Saddle nose deformity and ambiguous external ear anatomy, right lateral view. (C) Ambiguous external ear anatomy, left lateral view.

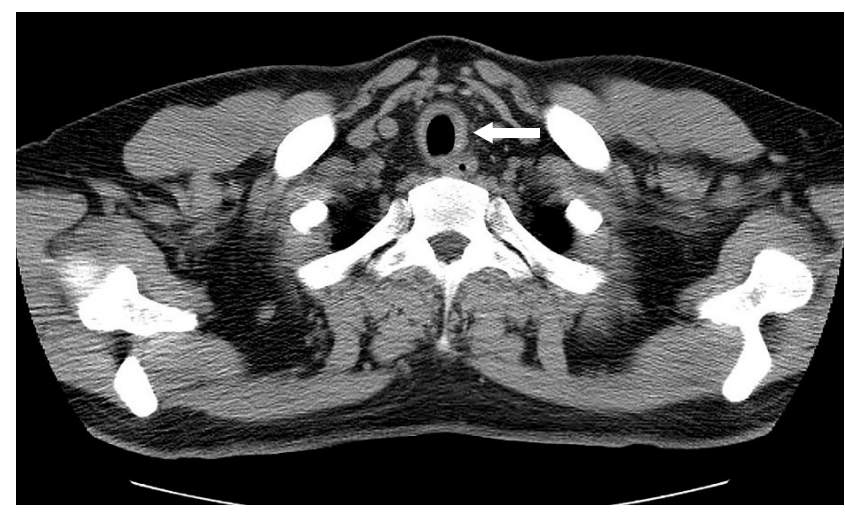

Fig. 2. Diffuse thickening of the proximal trachea (white arrow) on chest computed tomography.

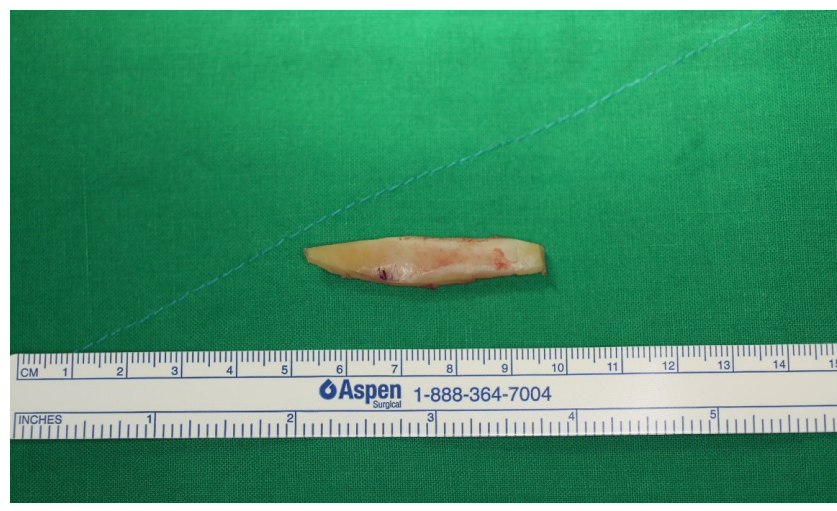

Fig. 3. The 8th costal cartilage carved to mimic the silicone implant. 


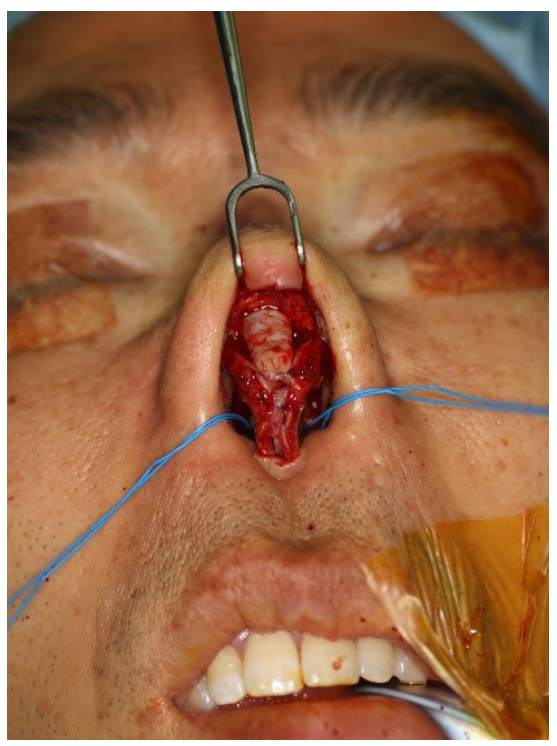

Fig. 4. Intraoperative clinical photograph.

and 4 years after the surgery). On the first episode, the patient complained of ocular pain and was diagnosed with episcleritis. On the second episode, the patient complained of diplopia and severe coughing. Both episodes were controlled with oral steroid therapy. Based on the medical records of the rheumatologist, throughout the two episodes of relapse, the patient had no complaints of change of neither nasal position and contour nor chest pain at the donor site.

The requirement for informed consent was waived, and this study was approved by the Institutional Review Board of our center (IRB No. 2019-07-058).

\section{DISCUSSION}

The surgical approach to correct the saddle nose deformity caused by RP has no established reasonable argument thus far. Due to its rare feature, only a few patients undergo rhinoplasty. There are also concerns regarding the viability of rhinoplasty in these patients as the surgery itself may accelerate the inflammatory response and break down the plateau status [6]. Therefore, the decision and timing of the surgery must be considered in the context of disease status and patient condition and is best planned in consultation with rheumatologists [7]. Surgery seems appropriate after immunological treatment or after the disorder has come to a stable remission [8].

Our patient underwent surgery when symptoms were stable even after cessation of oral steroid. The patient showed no relapse of RP over 15 months when the first episode of relapse occurred. After PSL treatment, the disorder showed stable remission for almost another 3 years.
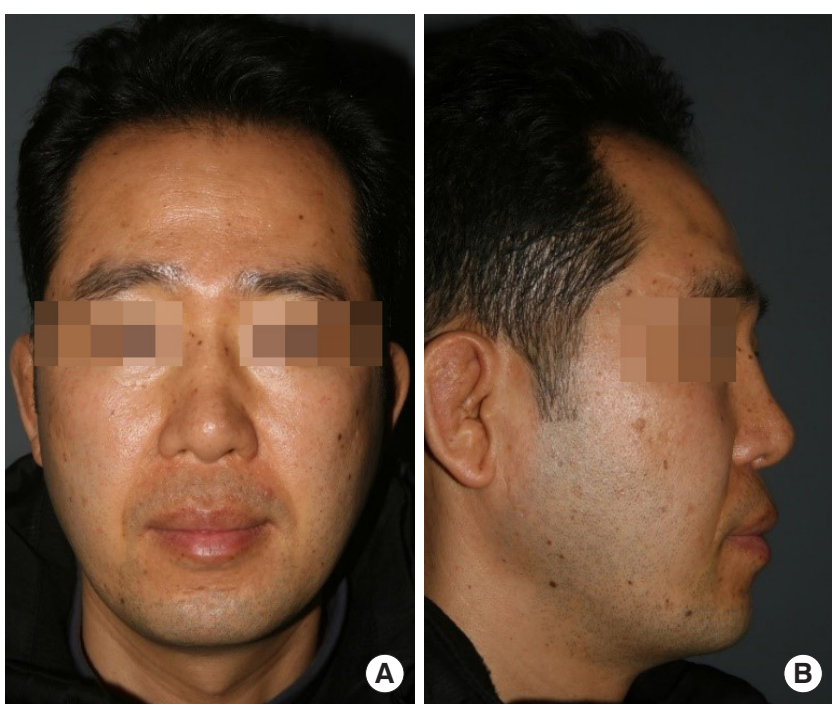

Fig. 5. Postoperative photographs at 6 months. (A) Anteroposterior view and (B) lateral view.

In previous cases reporting surgical treatment for saddle nose deformities caused by RP, silicone implants [9], calvarial bone grafts [10], iliac bone [2], and rib cartilage [8] were used to reconstruct the septum.

Autologous grafts are preferential to alloplastic implants because of their high biocompatibility and low risk of infection and extrusion [11]. In addition, the possibility that implants may raise a foreign body reaction leading to relapse of $\mathrm{RP}$ meant that alloplastic materials such as screws were not used for this case.

We selected the costal cartilage as the grafting material for several reasons. The relapsing characteristic of RP led us to believe that surgical treatment should not be aggressive as it can trigger the inflammation. In this regard, we needed a large-size autologous material that could be easily harvested. We decided that relatively easily obtained rib cartilage could be an option and costal cartilage seemed suitable to meet the demands of large defects. In addition, costal cartilage resembles nasal cartilage more than bone graft and is easier to mold and carve to the optimal shape, and considering the patients age and the final results for which we aimed, costal cartilage was used. Furthermore, as the patient had neither involvement from the costal cartilage nor history of chest pain, we decided at the time of surgery that the costal cartilage was not a contraindication for our case.

However, we do agree that this may not always be the case. In the study of Maciazek-Chyra et al. [12], one patient out of 10 (20\%) showed costal chondritis and Borgia et al. [1] also reported that involvement of costal cartilages occurs in 35\% of patients, but rare at the moment of diagnosis. This means that 
though our patient showed no signs of costal chondritis at diagnosis or time of surgery, it may occur within lifetime. However, to our knowledge there are no reports of costal chondritis after reconstruction with autologous costal cartilage grafting and uncertainty of the pathophysiology of RP makes it difficult to prevent this. Therefore, we should be cautious of chest wall pain or swelling to engage in an early stage to control the symptoms.

Few patients with RP undergo reconstructive rhinoplasty, which makes it difficult for us to prove that this would work out for all RP patients. However, the fact that the patient retained good esthetic results, showed no complication nor relapse of RP at 6-month follow-up, and still retained acceptable nasal shape and contour without donor site problems even after two relapse episodes of RP throughout the next 4 years, costal cartilage seems an option for reconstruction in RP patients with saddle nose deformity.

In conclusion, deliberate selection of donor graft must be beforehand any surgical procedure and we suggest the use of costal cartilage in reconstructive rhinoplasty for RP patients with saddle nose deformity, particularly among the relatively younger population without involvement of costal cartilage who seek optimal esthetic results.

\section{NOTES}

\section{Conflict of interest}

No potential conflict of interest relevant to this article was reported.

\section{Ethical approval}

The study was approved by the Institutional Review Board of Konkuk University Medical Center (IRB No. 2019-07-058) and performed in accordance with the principles of the Declaration of Helsinki.

\section{Patient consent}

The patient provided written informed consent for the publication and the use of his images.

\section{ORCID}

Yunhae Lee https://orcid.org/0000-0002-7801-8898

Hyungon Choi https://orcid.org/0000-0002-3816-1286

\section{REFERENCES}

1. Borgia F, Giuffrida R, Guarneri F, Cannavo SP. Relapsing polychondritis: an updated review. Biomedicines 2018;6:E84.

2. Haug MD, Witt P, Kalbermatten FD, Rieger UM, Schaefer DJ, Pierer G. Severe respiratory dysfunction in a patient with relapsing polychondritis: should we treat the saddle nose deformity? J Plast Reconstr Aesthet Surg 2009;62:e7-10.

3. McAdam LP, O’Hanlan MA, Bluestone R, Pearson CM. Relapsing polychondritis: prospective study of 23 patients and a review of the literature. Medicine (Baltimore) 1976;55:193-215.

4. Damiani JM, Levine HL. Relapsing polychondritis: report of ten cases. Laryngoscope 1979;89(6 Pt 1):929-46.

5. Isaak BL, Liesegang TJ, Michet CJ Jr. Ocular and systemic findings in relapsing polychondritis. Ophthalmology 1986;93:681-9.

6. Gubbels SP, Barkhuizen A, Hwang PH. Head and neck manifestations of Wegener's granulomatosis. Otolaryngol Clin North Am 2003;36:685-705.

7. Tobisawa Y, Shibata M. A case of saddle nose deformity caused by relapsing polychondritis: a long-term follow-up report after iliac bone grafting. J Plast Reconstr Aesthet Surg 2013;66:1621-2.

8. Bell D, Wright D, Witt PD. Durability of nasal reconstruction in an adolescent with relapsing polychondritis treated with infliximab. Plast Reconstr Surg 2007;120:1087-8.

9. Jones FR. Relapsing polychondritis: case report. Plast Reconstr Surg 1973;51:331-3.

10. Torossian JM, Dumas V, Allias F, Ninet J, Beziat JL. Nasal involvement in atrophic polychondritis: a case report. Ann Chir Plast Esthet 1999;44:262-5.

11. Sajjadian A, Rubinstein R, Naghshineh N. Current status of grafts and implants in rhinoplasty: part I. Autologous grafts. Plast Reconstr Surg 2010;125:40e-49e.

12. Maciazek-Chyra B, Szmyrka M, Skoczynska M, Sokolik R, Lasocka J, Wiland P. Relapsing polychondritis: analysis of symptoms and criteria. Reumatologia 2019;57:8-18. 